\title{
Diagnosis of Breast Cancer in Digital Mammograms Using Independent Component Analysis and Neural Networks
}

\author{
Lúcio F.A. Campos, Aristófanes C. Silva, and Allan Kardec Barros \\ Laboratory for Biologic Information Processing, \\ University of Maranhão, Av. dos portugueses, \\ s/n, Campus do Bacanga \\ lucio@dee.ufma.br, ari@dee.ufma.br, akbarros@ieee.org
}

\begin{abstract}
We propose a method for discrimination and classification of mammograms with benign, malignant and normal tissues using independent component analysis and neural networks. The method was tested for a mammogram set from MIAS database, and multilayer perceptron neural networks, probabilistic neural networks and radial basis function neural networks. The best performance was obtained with probabilistic neural networks, resulting in $97.3 \%$ success rate, with $100 \%$ of specificity and $96 \%$ of sensitivity.
\end{abstract}

Keywords: Mammogram, breast cancer, independent component analysis, neural networks, computer aided diagnosis.

\section{Introduction}

Breast cancer is the major cause of death by cancer in the female population. It is know that the best prevention method is early diagnosis, which lessens the mortality and enhances the treatment [1]. Therefore, a great effort has been made to improve the early diagnosis techniques. Among them, the most used is the mammogram, for it is low cost and easy access. However, mammogram has a high error value for medical diagnosis, ranging from 10 to $25 \%$, resulting in a great number of false-positives diagnostics, which causes unneeded biopsies, or false-negatives, which delays the cancer diagnosis. The medical diagnosis using mammography can be aided by image processing and computational vision algorithms, combined with artificial intelligence for features extraction. Those algorithms are able to decrease the error and make the mammograms more reliable [2].

The breast cancer is originated by an exaggerated and disordered cell multiplication, forming a lesion. This lesion is called malignant when its cells have the capacity to cause metastases, that is, invade other healthy cells around them. If those malignant cells reach the blood circulation, they could get into contact with other parts of the body, invading new cells and originate new tumors [3].

On the other hand, the benign lesions do not have this capacity. Their growth is slower, until a maximum fixed size, and they cannot spread to other organs. These kind of lesion is common in the breasts [4]. 
The mammography of benign tumors are well-defined, circular, with homogeneous texture. The malignant tumors, however, have speculated shape, frequently asymmetric, and less homogeneous than the benign lesions [5].

There are yet structures that can lead to medical misdiagnosis, as calcifications that arise as circular white spots [6].

CAD (Computer-Aided Diagnosis) systems can aid radiologists by providing a second opinion and may be used in the first stage of examination. For this to occur, it is important to develop many techniques to detect and recognize suspicious lesions and also to analyze and discriminate them. Some methods of lesion diagnosis in mammograms images have been reported. In [7], a system based in density-weighted contrast enhancement ( $D W C E$ ) was used, obtaining $82.33 \%$ of success. In [8] mammograms are classified by support vector machines (SVM). The system sensibility was $84 \%$. Christoyianni et al [9] compared three methods: Gray level histogram moments (GLHM), Spacial Gray Level Dependence Matrix (SGLD) and Independent Component Analysis (ICA). Accordingly to the authors, ICA had a better performance, with $88 \%$ of successful discrimination between normal and abnormal lesions, and $79.31 \%$ between benign and malignant lesions.

The proposed method is based on feature extraction by Independent Component Analysis. This technique is applied to many situations, as signal processing in cocktail-party environments [10], and pattern recognition in ECG and MEG [11], [12], [13].

Into this work, an image is taken as a linear combination of basis images, mutually statically independents, found using ICA. Such an basis image are extracted through the FastICA algorithm, from a preselected set of region of interest (ROI) of benign, malignant or normal tissues.

The objective of this work is to classify a ROI as normal, benign or malignant from the coefficients (features) extracted using ICA. Then, those features are used as input parameters to a Neural Network do the classification.

We divide this work as follows. Into section 2 we show the techniques for feature extraction and classification of ROI. In Section 3 we present the results and discuss about the application of the techniques under study. Finally, Section 4 presents some concluding remarks.

\section{Methods}

The block diagram of the proposed method is shown in figure 1. It consists of the selection of ROIs, the extraction of features using ICA, reduction of insignificants features using the forward-selection technica and the classification of the ROIs through neural networks.

\subsection{Independent Component Analysis}

Let us assume that an image is the sum of basis images $s_{1}, . ., s_{\mathrm{n}}$, mutually statistically independent. The image is then composed by the combination of $n$ basis images, where we have $n$ coefficients $a_{1} \ldots a_{\mathrm{n}}$ [9], [14], such that

$$
x_{i}=a_{i 1} \cdot s_{1}+a_{i 2} \cdot s_{2}+\ldots+a_{i n} \cdot s_{n} \quad \forall_{i}=1, \cdots n
$$




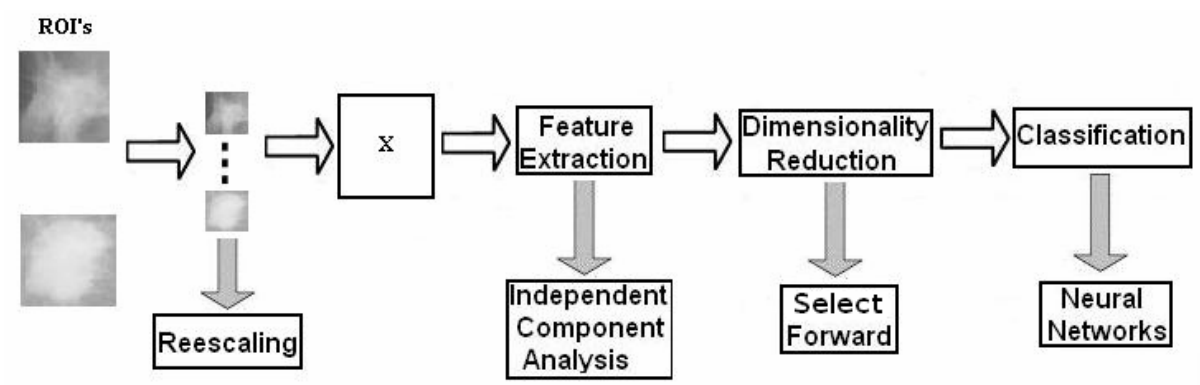

Fig. 1. Block diagram of the proposed method

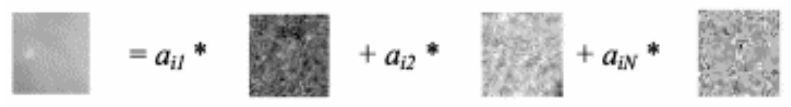

Fig. 2. Region of interest as a linear combination of its basis image mutually statistically independent

In equation 1 , only the variables $x_{i}$ are known, and from them we estimate the coefficients $a_{i j}$ and the independent components $s_{j}$, that is:

$$
X=A . S
$$

Where $\boldsymbol{X}$ is an mixture matrix, the columns of $\boldsymbol{A}$ are the basis functions and $\boldsymbol{S}$ are the basis images.

\subsubsection{FastICA Algorithm}

The FastICA algorithm is used to solve the blind source separation (BSS) problem, where we want to estimate the basis images and the basis functions of the image $\mathbf{X}$. This algorithm is based on fixed-point iteration [15], [16], [17]. In [15], a fixed-point algorithm was introduced using kurtosis, and in [16]-[17], the FastICA algorithm was generalized for general contrast functions [16]. For sphered data, the one-unit FastICA algorithm has the following form:

$$
w(k)=E\left\{x \cdot g\left(w(k-1)^{T} . x\right)\right\}-E\left\{g^{\prime}\left(w(k-1)^{T} . x\right)\right\} . w(k-1)
$$

Where the weight vector $\boldsymbol{w}$ is also normalized to unit norm after every iteration, and

$$
w^{-1}=A
$$

The function $g$ is the derivative of the function $G$ used in the general contrast function in equation 5 .

$$
J_{G(y)}=\left|E_{y}\{G(y)\}-E_{v}\{G(v)\}\right|^{p}
$$


Where $v$ is a standardized Gaussian random variable, $y$ is assumed to be normalized to unit variance, and the exponent $p=1,2$ typically. The subscripts denote expectation with respect to $y$ and $v$.

The basic form of the FastICa algorithm is as follows [14]:

1. Choose an initial (e.g. random) weight vector $\mathbf{w}$.

2. Let $w^{+}=E\left\{x g\left(w^{T} \cdot x\right)\right\}-E\left\{g^{\prime}\left(w^{T} x\right)\right\}^{w} w$

3. Let $w=w^{+} /\left\|w^{+}\right\|$

4. If not converged, go back to 2 .

The expectations are estimated, in practice, using sample averages over a sufficiently large sample of the input data. Units using this FastICA algorithm can then be combined, just as in the case of neural learning rules, into systems that estimate several independent components. Such systems may either estimate the independent component one-by-one using hierarchical decorrelation (deflation), or they may estimate all the independent components [16]-[17].

\subsection{Neural Networks}

In this work, we use a Multilayer Perceptron Neural Network (MLP), Probabilistic Neural Network (PNN) and Radial Basis Functions Neural Network (RBFNN) to classify malignant, benign and normal tissues.

\subsubsection{Multilayer Perceptron Neural Networks}

The Multilayer Perceptron (MLP), a feed-forward back-propagation network, is the most frequently used neural network technique in pattern recognition [18], [19].

Speaking, MLPs are supervised learning classifiers that consist of an input layer, an output layer, and one or more hidden layers that extract useful information during learning and assign modifiable weighting coefficients to components of the input layers. In the first (forward) pass, weights assigned to the input units and the nodes in the hidden layers and between the nodes in the hidden layer and the output, determine the output. The output is compared with the target output. An error signal is then back propagated and the connection weights are adjusted correspondingly. During training, MLPs construct a multidimensional space, defined by the activation of the hidden nodes, so that the three classes (malignant, benign and normal tissue) are as separable as possible. The separating surface adapts to the data.

\subsubsection{Probabilistic Neural Network}

The probabilistic neural network (PNN) is a direct continuation of the work on Bayes classifiers. The PNN learns to approximate the $p d f$ of the training examples [19].

More precisely, the PNN is interpreted as a function which approximates the probability density of the underlying example

The PNN consists of nodes allocated in three layers after the inputs:

- pattern layer: there is one pattern node for each training example. Each pattern node forms a product of the weight vector and the given example for classification, 
where the weights entering a node are from a particular example. After that, the product is passed through the activation function:

$$
\exp \left[\left(\boldsymbol{x}^{T} \boldsymbol{w}_{k i}-1\right) / \sigma^{2}\right]
$$

Where

- $\quad \mathrm{x}$ : Data input

- $\quad \mathrm{W}_{\mathrm{k}}$ : Weight

- $\sigma:$ Smothing adjust

- summation layer: each summation node receives the outputs from pattern nodes associated with a given class:

$$
\Sigma_{i=1}^{N k} \exp \left[\left(\mathbf{x}^{\mathrm{T}} \mathbf{w}_{k i}-1\right) / \sigma^{2}\right]
$$

- output layer: the output nodes are binary neurons that produce the classification decision

$$
\sum_{i=1}^{N k} \exp \left[\left(\mathbf{x}^{\mathrm{T}} \mathbf{w}_{k i}-1\right) / \sigma^{2}\right]>\Sigma_{i=1}^{N j} \exp \left[\left(\mathbf{x}^{\mathrm{T}} \mathbf{w}_{k j}-1\right) / \sigma^{2}\right]
$$

\subsubsection{Radial Basis Functions Neural Networks}

Successful implementation of the Radial Basis Functions Neural Network (RBFNN) can be achieved using efficient supervised or unsupervised learning algorithms for an accurate estimation of the hidden layer [20]-[21].

In our implementation, the k-means unsupervised algorithm was used to estimate the hidden layer weights from a set of training data containing the features from malignant, benign and normal tissue. After the initial training and the estimation of the hidden layer weights, the weights in the output layer are computed using Wienerfilter, for example, by minimizing the mean square error (MSE) between the actual and the desired output over the set of samples.

The RBFNN have a faster learning rate and have been proved to provide excellent discrimination in many applications.

\subsection{Selection of Most Significant Features}

Our main objective is to identify the effectiveness of a feature or a combination of features when applied to a neural network. Thus, the choice of features to be extracted is important.

Forward selection is a method to find the "best" combination of features (variables) by starting with a single feature, and increasing the number of used features, step by step [22]. In this approach, one adds features to the model one at a time. At each step, each feature that is not already in the model is tested for inclusion in the model. The most significant of these feature is added to the model, so long as P-value is below some pre-selected level. 


\subsection{Evaluation of the Classification Method}

Sensitivity and specificity are the most widely used statistics to describe a diagnostic test. Sensitivity is the proportion of true positives that are correctly identified by the test and is defined by $\boldsymbol{S}=\boldsymbol{T P} /(\boldsymbol{T P}+\boldsymbol{F N})$. Specificity is the proportion of true negatives that are correctly identified by the test and is defined by $\boldsymbol{T N} /(\boldsymbol{T N}+\boldsymbol{F P})$. Where $\mathbf{F N}$ is false-negative, $\mathbf{F P}$ is false-positive, $\mathbf{T N}$ is true negative and $\mathbf{T P}$ is true positive diagnosis.

\section{Experimental Results and Discussions}

Here are describe the results obtained using the method proposed in the previous section.

\subsection{Mammogram Database}

The database used into this work is the Mammographic Institute Society Analisys (MIAS) [23]. The mammograms have a size of 1024 x 1024 pixels, and resolution of 200 micron. This database is composed of 332 mammograms of right and left breast, from 161 patients, where 53 were diagnosed as being malignant, 69 benign and 206 normal. The abnormalities are classified by the kind of found abnormality (calcification, circumscribed masses, architectural distortions asymmetries, and other illdefined masses) .

This database contains a file lists the mammograms in the MIAS database and provides appropriate details, for example, the class of abnormality, xy image-coordinates of centre of abnormality, and approximate radius (in pixels) of a circle enclosing the abnormality.

From this database, we selected 100 abnormal ( 50 benign and 50 malignant mammograms) and 100 normal from each group, summing up 200 mammograms. To each mammogram, a ROI was manually selected, containing the lesion, in the case of the benign and malignant mammograms. The ROIs was found through of xy images coordinates of centre of abnormality, contained in file list of MIAS database . To the normal mammograms, was randomly selected the ROI. Only the pectoral muscle was not considered as a possible ROI, although tissue and fatty tissue were. If the tissues had different sizes, it was rescaled each ROI. Therefore, they were resized to $24 \times 24$ pixels. Figure 3 exemplifies the ROI selection of a mammogram diagnosed as benign, malignant and normal, respectively.

\subsection{ICA Application}

$X$ of Equation 2 was represented using the chosen ROIs. The images with ROI were rescaled and transformed into a one-dimensional vector

$$
P=P_{x} x P_{y}
$$

where $\boldsymbol{P}_{\boldsymbol{x}}$ is a rows and $\mathbf{P}_{\mathbf{y}}$ is a colunns of $\boldsymbol{P}$ and $\boldsymbol{P}$ has dimension $1 \mathrm{x} 576$. 


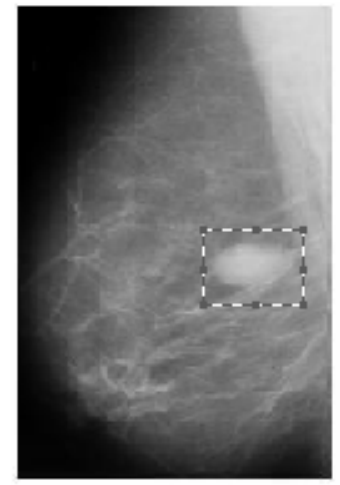

(a)

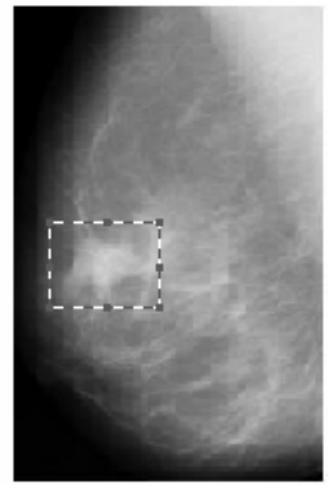

(b)

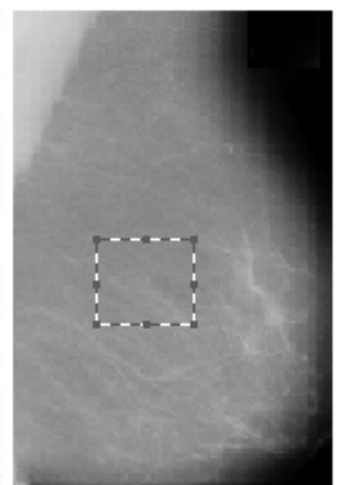

(c)

Fig. 3. Chosen region of interest (ROI) of mammograms diagnosed as benign (a), malignant (b) and normal (c)

Each sample represents one row of the mixture matrix. The matrix $\mathbf{X}$ is represented by the samples into the dimension of $\mathbf{P}$, that is, $1 \times 576$. Thus, each row of the matrix $\mathbf{A}$ correspond to a ROI, and each column correspond to an attributed weight to a base image, i.e., an input parameter to the neural network [9].

Using the FastICA algorithm and the matrix $\boldsymbol{X}$, we obtain the basis function matrix $\mathbf{A}$, which contains the features of each sample.

Figure 4 exemplifies the basis image found using the basis functions of the malignants, benigns and normal ROIs, respectively. We can clearly observe the differences between the basis images of each kind. Thus, the basis functions of the benign tissue are different of those of the malignant tissue.

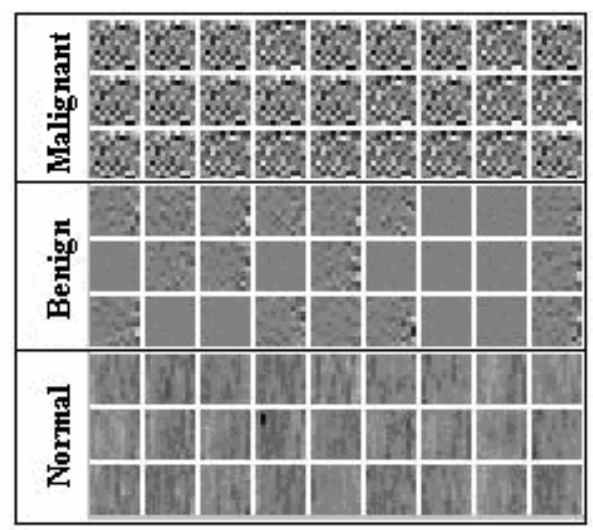

Fig. 4. Basis image sample produced from the ROIs basis functions for normal, benign and malignant tissue 


\subsection{Neural Networks}

Using the forward-selection algorithm, basis functions were selected as being the most significant features. The chosen features $\left(a_{i}\right)$ are the input to the Neural Network. For each Neural Networks (MLP, PNN, RBFNN) the algorithm selected the most significant features.

We carried out tests with different Neural Network architetures to find the bests MLP, PNN and RBF Neural Networks.

In order to carry out the tests, we divided a sample in 200 ROIs: 100 for training and 100 for tests.

\subsection{Results}

Table 1 shows the Neural Networks architecture, the performance of the application of the ICA technique with each Neural Network for discrimination tissues (ROI).

Table 1. Neural Networks architecture and classification of malignant, benign and normal ROI

\begin{tabular}{ccccccccc}
\hline \multirow{2}{*}{ N.Networks } & Arquiteture & TP & TN & FP & FN & \multicolumn{4}{c}{$(\%)$} \\
\hline PNN & $50: 76-3: 1$ & 96 & 10 & 0 & 4 & 100 & 96 & 97.3 \\
RBF & $44: 15-3: 1$ & 93 & 98 & 2 & 7 & 98 & 93 & 94.6 \\
MLP & $44: 21: 3$ & 90 & 98 & 2 & 9 & 98 & 90.91 & 92.6 \\
\hline
\end{tabular}

Based on the Table 1, the best results was obtained with Probabilistic Neural Networks. The PNN obtained a success rate of $97.3 \%$ on discriminating malignant, benign and normal tissues. The found specificity was $100 \%$ and the sensitivity, $96 \%$. The PNN obtained 96 true positives diagnosis, 100 true negatives, 0 false positives and 4 false negatives.

\section{Conclusion}

The presented results demonstrate that Independent Component Analysis and Neural Networks is a useful tool to discriminate malignant, benign and normal tissues.

Furthermore, the Probabilistic Neural Network obtained the best performance, classifying those tissues, with a success rate of $97.3 \%$, specificity of $100 \%$ and sensitivity of $96 \%$. It can decrease the number of unneeded biopsies and late cancer diagnosis.

Based on these results, we have observed that such features provide significant support to a more detailed clinical investigation, and the results were very encouraging when tissues were classified with ICA and Neural Networks. 


\section{Acknowledgements}

To all my co-workers in the Laboratory for Biological Information Processing, specially Denner Guilhon, André Cavalcante and Fausto Lucena.

\section{References}

1. INCa, Internet site address: http://www.inca.gov.br/acessed in 04/05/2005.

2. U. Bick, M. Giger, R. Schmidt, R. Nishikawa, D. Wolverton and K. Doi.Computer- aided breast cancer detection in screening mammography. Digital Mammogr'9Chicago, IL (1996), pp. 97-103.

3. J. G. Elmore, C. K. Wells, C. H. Lee, D. H. Howard, and A. R. Feinstein, IVariability in radiologists' interpretations of mammograms," New England Journal of Medicine, vol. 331, no. 22, pp. 1493 \{1499, December 1994.

4. L. W. Bassett, V. P. Jackson, R. Jahan, Y. S. Fu, and R. H. Gold, Diagnosis of Diseases of the Breast. W. B. Saunders Company, 1997.

5. D. B. Kopans, The positive predictive value of mammography," American Journal of Roentgenology, vol. 158, no. 3, pp. 521\{526, March 1993.

6. L. Tabar and P. B. Dean, Teaching Atlas of Mammography. Georg ThiemeVerlag, 2nd revised ed., 1985.

7. Nicholas Petrick, Berkman Sahiner, HeangPing Chan, Mark A. Helvie, Sophie Paquerault, and Lubomir M. Hadjiiski. Breast Cancer Detection: Evaluation of a MassDetection Algorithm for Computer-aided Diagnosis-Experience in 263 Patients Radiology 2002;224:217-224.

8. Campanini Renato, Bazzani Armando, et al. A novel approach to mass detection in digital mammography based on Support Vector Machines (SVM). In proceedings of the $6^{\text {th }}$ International workshop in digital Mammography (IWDM), pages 399-401, Bremem, Germany, 2002, Springer Verlag.

9. Christoyianni I., Koutras A., Kokkinakis G., "Computer aided diagnosis of breast cancer in digitized mammograms", Comp. Med. Imag. \& Graph., 26:309-319, 2002.

10. B. Arons, A review of cocktail party, Cambridge, MA: MIT laboratory, 1990

11. R. Vigário, Extraction of ocular artifacts form ecg using independent components analysis, Eletroenceph. Clin. Neurophysiol., 103 (3) : 395-404, 1997

12. R.vigário. V. Jousmaki, M. Hamalaien R. Hari, and E. Oja, Independent component analysis for identification of artifacts in magnetoencephalographic recordings, In andvances in neural information processing 10 (Proc. NIPS'97), 229-235, Cambridge, Ma, MIT press, 1998

13. S. Makeig, A. J. Bell, T-P. Jung, and T.J. Sejnowski, Indepent component analysis of electroencephalographic data, In Advances in neural information processing systems 8, 145151. MIT press, 1996.

14. Hyvärinen, A., J. Karhunen, and E. Oja, Independent Component Analysis, John Wiley \& Sons, New York, 2001.

15. A. Hyvärinen and E. Oja. A fast fixed-point algorithm for independent component analysis. Neural Computation, 9(7):1483-1492, 1997. 
16. A. Hyvärinen. A family of fixed-point algorithms for independent component analysis. In Proc. IEEE Int. Conf. on Acoustics, Speech and Signal Processing (ICASSP'97), pages 3917-3920, Munich, Germany, 1997.

17. A. Hyvärinen. Fast and robust fixed-point algorithms for independent component analysis. IEEE Trans. on Neural Networks, 1999.

18. Duda, R.O., Hart, P.E.: Pattern Classification and Scene Analysis. WileyInterscience Publication, New York (1973)

19. Bishop, C.M.: Neural Networks for Pattern Recognition. Oxford UniversityPress, New York (1999)

20. Christoyianni I, Dermatas E, Kokkinakis G. Fast detection of masses in computer-aided mammography. IEEE Signal Process Mag 2000; 17(1):54-64.

21. Christoyianni I, Dermatas E, Kokkinakis G. Neural classification of abnormal tissue in digital mammography using statistical features of the texture. IEEE Int Conf Electron, Circuits Syst 1999;1:117-20.

22. Fukunaga, K.: Introduction to Statistical Pattern Recognition. 2 edn. AcademicPress, London (1990)

23. J Suckling et al (1994): The Mammographic Image Analysis Society Digital Mammogram Database Exerpta Medica. International Congress Series 1069 pp375-378. 